\title{
Willingness to Wait and Altered Encoding of Time-Discounted Reward in the Orbitofrontal Cortex with Normal Aging
}

\author{
Matthew R. Roesch, ${ }^{1,2}$ Daniel W. Bryden, ${ }^{1,2}$ Domenic H. Cerri, ${ }^{3}$ Zachary R. Haney, ${ }^{3}$ and Geoffrey Schoenbaum ${ }^{4}$ \\ ${ }^{1}$ Department of Psychology and 2 Program in Neuroscience and Cognitive Science, University of Maryland College Park, College Park, Maryland 20742, \\ ${ }^{3}$ Department of Anatomy and Neurobiology, University of Maryland School of Medicine, Baltimore, Maryland 21201, and ${ }^{4}$ NIDA Intramural Research \\ Program, Baltimore, Maryland 21224
}

\begin{abstract}
Normal aging has been associated with cognitive changes, including shifts in responding for time-discounted rewards. The orbitofrontal cortex, an area previously associated with aging-related cognitive changes, is critical for normal discounting. Previously we have shown in a choice task that rats prefer immediate over delayed reward and that neural representations of delayed reward in orbitofrontal cortex were attenuated, whereas immediate reward elicited strong responses. Changes in choice performance were correlated with changes in firing rate in orbitofrontal neurons, suggesting that these reward representations were critical to the rats' ability to wait for reward. Here we asked whether age-dependent changes in discounting behavior were related to changes in the representation of delayed reward in the orbitofrontal cortex. Young (3-6 months) and aged (22-26 months) rats were trained on the same discounting paradigm used previously. We found that aged rats showed less sensitivity to increasing delay preceding reward delivery, shifting behavior away from the delayed reward more slowly than younger rats. This sensitivity was specific to delay, since choice performance did not differ between the two groups when delay was held constant and reward size varied. Aged rats exhibited a corresponding increase in the prevalence of neurons that fired more strongly for delayed reward. Again this change was specific to delay; there was no change in encoding of different-sized rewards. These results suggest that natural aging results in altered representations of reward in orbitofrontal cortex. These changes may relate to the increased ability to delay gratification and reduced impulsivity associated with aging.
\end{abstract}

\section{Introduction}

Normal aging, distinct from pathological entities associated with frank dementia, is associated with reduced impulsivity and a greater ability to forgo short-term benefits in favor of more distant gratification (Simon et al., 2010). In experimental settings, the ability to wait for delayed rewards is typically assessed in so-called discounting tasks in which an immediate small reward is pitted against a larger delayed reward (Herrnstein, 1961; Rachlin and Green, 1972; Ainslie, 1974; Evenden and Ryan, 1996). In rats, the neural system mediating discounting includes the nucleus accumbens (NAc), basolateral amygdala (BLA), and orbitofrontal cortex (OFC) (Cardinal et al., 2001; Mobini et al., 2002; Winstanley et al., 2004; Rudebeck et al., 2006; Bezzina et al., 2007; Floresco et al., 2008; Kalenscher and Pennartz, 2008; Churchwell et al., 2009; Ghods-Sharifi et al., 2009; Sellitto et al., 2010; Mar et

Received Feb. 7, 2012; revised Feb. 28, 2012; accepted March 2, 2012.

Author contributions: M.R.R. and G.S. designed research; M.R.R., D.H.C., Z.R.H., and G.S. performed research; M.R.R., D.W.B., and G.S. analyzed data; M.R.R. and G.S. wrote the paper.

This work was supported by grants from NIA to G.S. and NIDA to M.R.R. This article was prepared in part while G.S. was employed at University of Maryland School of Medicine, Baltimore. The opinions expressed in this article are the author's own and do not reflect the view of the National Institutes of Health, the Department of Health and Human Services, or the United States government.

Correspondence should be addressed to either of the following, at the above addresses: Geoffrey Schoenbaum, E-mail: schoenbg@schoenbaumlab.org; or Matthew R. Roesch, E-mail: mroesch@umd.edu.

DOI:10.1523/JNEUROSCI.0586-12.2012

Copyright $\odot 2012$ the authors $\quad 0270-6474 / 12 / 325525-09 \$ 15.00 / 0$ al., 2011). Within this circuit, the OFC seems particularly crucial to the normal representation of the delay. For example, the OFC is not normally necessary for distinguishing rewards based on magnitude or cost (Winstanley et al., 2004; Rudebeck et al., 2006; McDannald et al., 2011), whereas the NAc and BLA are often required for normal behavior in settings that test for these variables (Salinas et al., 1993; Kesner and Williams, 1995; Cousins et al., 1996; Ghods-Sharifi et al., 2009; Ghods-Sharifi and Floresco, 2010; McDannald et al., 2011).

Consistent with this idea, neural activity in the NAc integrates information about size, timing, or cost of rewards (Roesch et al., 2009; Day et al., 2010, 2011; Cai et al., 2011), whereas this is typically not the case in the OFC (Roesch et al., 2006). For example, we reported two types of neurons in the OFC; one that fired more strongly to immediate reward compared with delayed reward and another that showed the opposite effect, firing more strongly for rewards that were delivered after a long delay. Activity of neurons of the latter type increased as reward delivery approached, and thus exhibited stronger firing when rewards were delayed. Such activity could influence choices between small immediate and large delayed rewards. Consistent with this idea, the activity of these discounting neurons correlated with the rats' ability to wait for the delayed reward.

The prefrontal cortex, and the OFC in particular, has been implicated in aging-related changes in decision-making in a variety of contexts (Rapp and Heindel, 1994; Zyzak et al., 1995; 


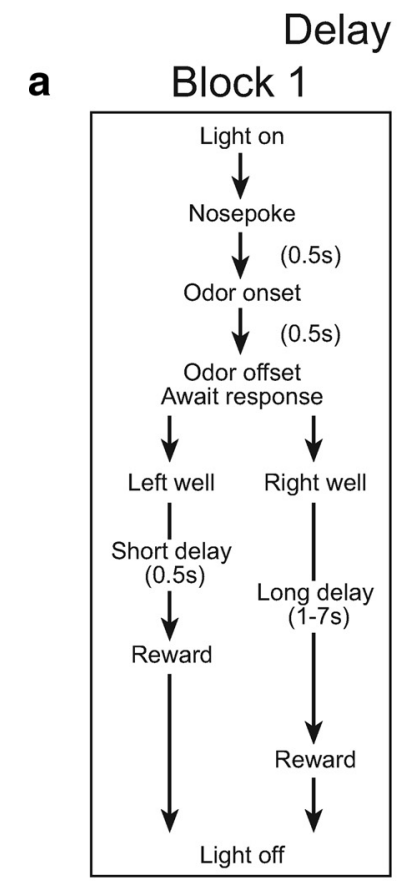

Blocks
Block 2

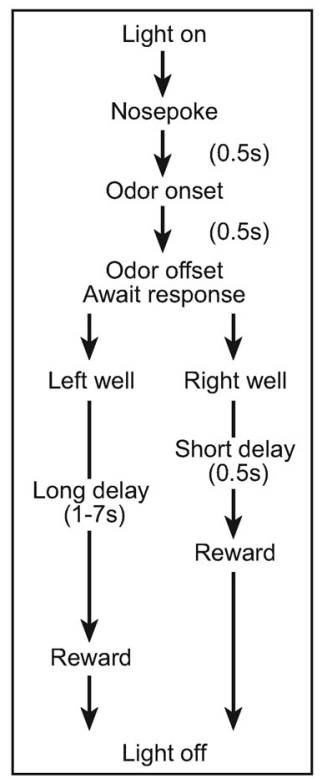

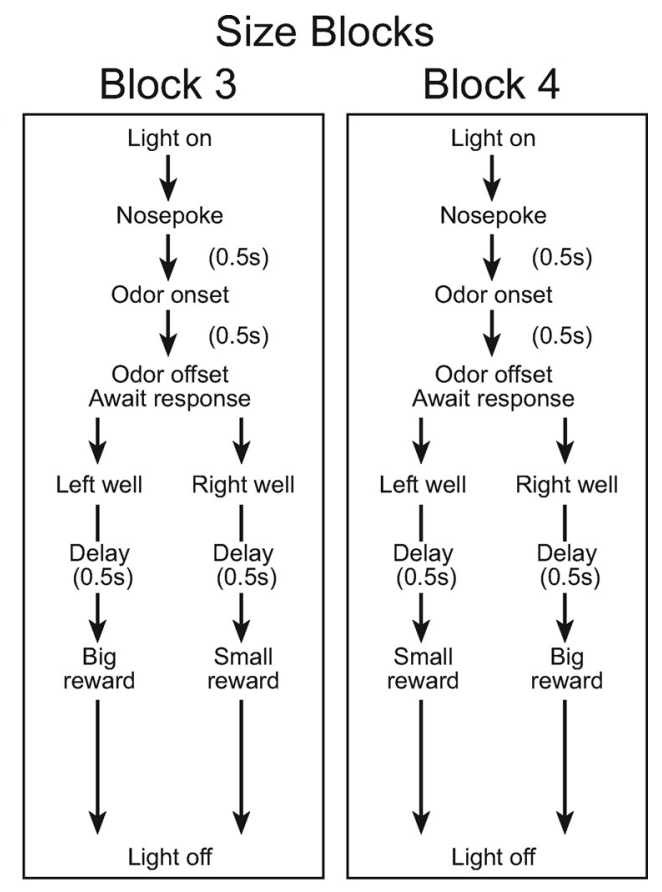

\section{Size Blocks}

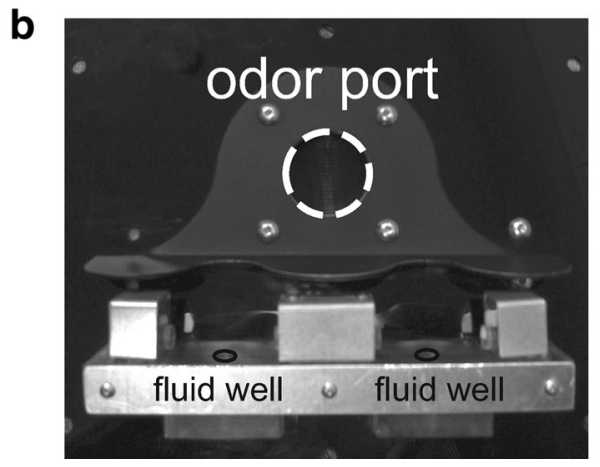

Figure 1. Behavioral Task. $\boldsymbol{a}$, Choice task during which we varied the delay preceding reward delivery and the size of reward. Figure shows sequence of events in each trial in 4 blocks in which we manipulated the time to reward or the size of reward. Trials were signaled by illumination of the panel lights inside the box. When these lights were on, nosepoke into the odor port resulted in delivery of the odor cue to a small hemicylinder located behind this opening. One of three different odors was delivered to the port on each trial, in a pseudorandom order. At odor offset, the rat had 3 s to make a response at one of the two fluid wells located below the port. One odor instructed the rat to go to the left to get reward, a second odor instructed the rat to go to the right to get reward, and a third odor indicated that the rat could obtain reward at either well. One well was randomly designated as short and the other long at the start of the session (block 1 ). In the second block of trials these contingencies were switched (block 2). In later blocks (3-4) we held the delay preceding reward delivery constant while manipulating the size of the expected reward. $\boldsymbol{b}$, Behavioral apparatus. A central odor port was located above and between two adjacent fluid wells on a panel in the right wall of each chamber.

Rypma and D'Esposito, 2000; Barense et al., 2002; Lamar et al., 2004; Smith et al., 2004; Gazzaley et al., 2005; Marschner et al., 2005; Moore et al., 2006; Brushfield et al., 2008; Lasarge et al., 2009; Mizoguchi et al., 2009; Wang et al., 2011). For example, aged rats are slower to acquire reversals, and these impairments have been linked to changes in processing in the OFC (Schoenbaum et al., 2002, 2006). More recently, it has been shown that aged rats exhibited attenuated discounting of delayed rewards (Simon et al., 2010). Here we tested whether these changes might also be linked altered neural processing in OFC.

\section{Materials and Methods}

Subjects. Male Long-Evans rats in the aged group $(n=6)$ were acquired at $\sim 10$ months of age (Charles River Laboratories) and housed for $\sim 1$ year in preparation for the experiment. During this time they were handled weekly. Testing began when they ranged from 22 to 24 months of age. Young controls $(n=6)$ were acquired at $\sim 2-3$ months of age $\sim 2$ weeks before testing (Charles River Laboratories). During this time they were handled daily. Rats were tested at the University of Maryland School of Medicine in accordance with School of Medicine and NIH guidelines.
Surgical procedures and histology. Surgical procedures followed guidelines for aseptic technique. Electrodes were manufactured and implanted as in prior recording experiments (Roesch et al., 2006). Rats had a drivable bundle of ten $25-\mu$ m-diameter FeNiCr wires (Stablohm 675, California Fine Wire) chronically implanted in the left hemisphere dorsal to OFC ( $3.0 \mathrm{~mm}$ anterior to bregma, $3.2 \mathrm{~mm}$ laterally, and $4.0 \mathrm{~mm}$ ventral to the brain surface; see Fig. $3 a$ ). Immediately before implantation, these wires were freshly cut with surgical scissors to extend $\sim 1 \mathrm{~mm}$ beyond the guide cannula and electroplated with platinum $\left(\mathrm{H}_{2} \mathrm{PtCl}_{6}\right.$, Aldrich) to an impedance of $\sim 300 \mathrm{k} \Omega$. Cephalexin $(15 \mathrm{mg} / \mathrm{kg}$, p.o.) was administered twice daily for 2 weeks postoperatively to prevent infection. Young and aged rats were approximately 4 and 24 months old, respectively, at the time of surgery.

Behavioral task. Recording was conducted in aluminum chambers $\sim 18$ inches on each side with sloping walls narrowing to an area of $12 \times$ 12 inches at the bottom. A central odor port was located above and between two adjacent fluid wells on a panel in the right wall of each chamber (Fig. 1b). Two lights were located above the panel. The odor port was connected to an air flow dilution olfactometer to allow the rapid delivery of olfactory cues. Task control was implemented via computer. Port entry and licking was monitored by disruption of photobeams. 


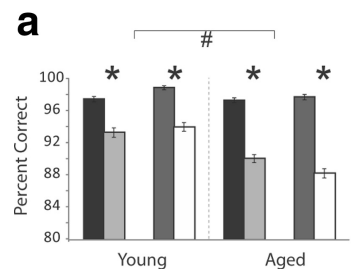

b

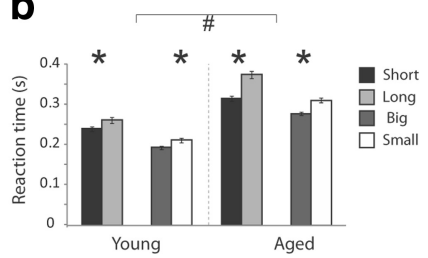

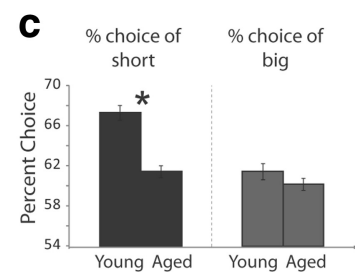

Figure 2. Behavior. $\boldsymbol{a}, \boldsymbol{b}$, Height of each bar indicates the percentage correct and reaction time (port exit minus odor offset) for short (black), long (light gray), big (dark gray), and small (white) forced-choice trials for young and aged rats. c, The height of each bar indicates the percentage choice of short delay versus long delay and big reward versus small reward (bottom) taken over all free-choice trials Asterisks: planned comparisons revealing statistically significant differences ( $t$ test, $p<0.05$ ). Error bars indicate SEs.

The basic design of a trial is illustrated in Figure $1 a$. Trials were signaled by illumination of the panel lights inside the box. When these lights were on, nosepoke into the odor port resulted in delivery of the odor cue to a small hemicylinder located behind this opening. One of three different odors was delivered to the port on each trial, in a pseudorandom order. At odor offset, the rat had $3 \mathrm{~s}$ to make a response at one of the two fluid wells located below the port. One odor (Verbena Oliffac) instructed the rat to go to the left to get reward, a second odor (Camekol DH) instructed the rat to go to the right to get reward, and a third odor (Cedryl Acet Trubek) indicated that the rat could obtain reward at either well. Odors were presented in a pseudorandom sequence such that the freechoice odor was presented on 7 of 20 trials and the left/right odors were presented in equal numbers ( \pm 1 over 250 trials). In addition, the same odor could be presented on no more than 3 consecutive trials. Odor identity did not change over the course of the experiment. Once the rats were shaped to perform this basic task, we introduced blocks in which we independently manipulated the size of the reward delivered at a given side or the length of the delay preceding reward delivery. Whereas ideal choice responses could net the animal more total reward during size blocks, responses had no influence on total reward during delay blocks. Once the rats were able to maintain accurate responding through these manipulations, we began recording sessions. For recording, one well was randomly designated as short (500 ms) and the other long (1-7 s) at the start of the session (Fig. 1a, block 1). In the second block of trials these contingencies were switched (Fig. 1a, block 2). The length of the delay under long conditions abided the following algorithm. The side designated as long started off as $1 \mathrm{~s}$ and increased by $1 \mathrm{~s}$ every time that side was chosen until it became $7 \mathrm{~s}$. If the rat chose the side designated as long $<8$ of the last 10 choice trials, then the delay was reduced by $1 \mathrm{~s}$ to a minimum of $3 \mathrm{~s}$. The reward delay for long forced-choice trials was yoked to the delay in free-choice trials during these blocks. In later blocks we held the delay preceding reward delivery constant $(500 \mathrm{~ms})$ while manipulating the size of the expected reward (Fig. $1 a$, blocks 3 and 4). All trials were balanced to last for the same total duration regardless of choice response. The reward was a $0.05 \mathrm{ml}$ bolus of $10 \%$ sucrose solution. For big reward, an additional bolus was delivered after $500 \mathrm{~ms}$. At least 60 trials per block were collected for each neuron. Rats were water deprived ( $\sim 30 \mathrm{~min}$ of free water at the end of the day) with free access on weekends. Rats were on a $12 \mathrm{~h}$ light/dark cycle and tested during the day.

Single-unit recording. Procedures were the same as described previously (Roesch et al., 2006). Young and aged rats were recorded from in an interleaved fashion over the course of 2-3 months. Wires were screened for activity daily; if no activity was detected, the rat was removed from the recording chamber, and the electrode assembly was advanced 40 or 80 $\mu \mathrm{m}$. Otherwise active wires were selected to be recorded, a session was conducted, and the electrode was advanced at the end of the session. Neural activity was recorded using two identical Plexon Multichannel Acquisition Processor (MAP) systems, interfaced with odor discrimination training chambers. Signals from the electrode wires were amplified $20 \times$ by an op-amp headstage located on the electrode array. Immediately outside the training chamber, the signals were passed through a differential preamplifier, where the single-unit signals were amplified $50 \times$ and filtered at $150-9000 \mathrm{~Hz}$. The single-unit signals were then sent to the MAP box, where they were further filtered at $250-8000 \mathrm{~Hz}$, digitized at
$40 \mathrm{kHz}$, and amplified at $1-32 \times$. Waveforms $(>2.5: 1$ signal-to-noise) were extracted from active channels and recorded to disk by an associated workstation with event timestamps from the behavior computer. Waveforms were not inverted before data analysis.

Data analysis. Units were sorted using Offline Sorter software from Plexon Inc, using a template-matching algorithm. Sorted files were then processed in Neuroexplorer to extract unit timestamps and relevant event markers. These data were subsequently analyzed in Matlab (MathWorks). Waveforms were not inverted. Waveform duration was measured from the maximum amplitude before to the maximum amplitude after the negative deflection. To examine activity related to reward delivery, we examined activity during the $1 \mathrm{~s}$ interval following reward delivery (reward epoch). To examine activity related to the processing of odors we examined activity during odor onset to odor port exit (odor epoch). Wilcoxon tests were used to measure significant shifts from zero in distribution plots $(p<$ $0.05)$. T tests or ANOVAs were used to measure within cell differences in firing rate $(p<0.05)$. Pearson $\chi^{2}$ tests $(p<0.05)$ were used to compare the proportions of neurons.

\section{Results}

Rats were trained on the choice task illustrated in Figure 1a. On each trial, rats responded to one of two adjacent wells after sampling an odor at a central port. Rats were trained to respond to three different odor cues: one odor that signaled reward in the right well (forced-choice), a second odor that signaled reward in the left well (forced-choice), and a third odor that signaled reward at either well (free-choice). Across blocks of trials we manipulated value by increasing the length of the delay preceding reward delivery (Fig. 1a, block 1-2) or by increasing the number of rewards delivered (Fig. 1a, block 3-4).

Both groups performed well on the task, although aged rats were slower to respond on forced choice trials (Fig. 2b; ANOVA; main effect of age; $p<0.0001$ ) and exhibited a slightly lower performance on these trials than young rats (Fig. $2 a$; ANOVA; main effect of age; $p<0.01)$. Both groups were significantly faster and more accurate on short-delay and large-reward trials compared with long-delay and small-reward trial types [Fig. $2 a, b$; ANOVA; main effect of value (size or delay); $p$ values $<0.01$ ]. Finally, the differences between high and low value measures of percent correct and reaction time on forced choice trials were more pronounced in the aged group as exhibited by a significant interaction (value $\times$ age; ANOVA; $p$ values $<0.05$ ) for all comparisons except for reaction times obtained during size blocks (value $\times$ age; ANOVA; $p=0.28$ ). Aged rats also did not exhibit any gross changes in the time spent to consume reward. Thus there was no significant difference between aged and young rats in the time spent in the well following reward delivery ( $t$ test; $p=$ $0.11)$. On average aged and young rats spent $5.19 \mathrm{~s}$ and $5.11 \mathrm{~s}$ in the well after delivery of reward, respectively.

Interestingly, aged rats did exhibit a delay-specific change in their response to the reward manipulations on free-choice trials; while both groups chose shorter delays and larger rewards over their respective counterparts (Fig. $2 c$; $t$ test; $p$ values $<0.0001$ ), aged rats chose to wait for the delayed reward significantly more often than younger rats (Fig. $2 c ; t$ test; $p<0.0001$ ). By contrast, the influence of reward size on free-choice performance did not differ significantly between groups (Fig. $1 c ; t$ test; $p=0.62$ ). Thus both groups were able to perceive differences in reward size and delay, as evidenced by differential performance measures on 
a
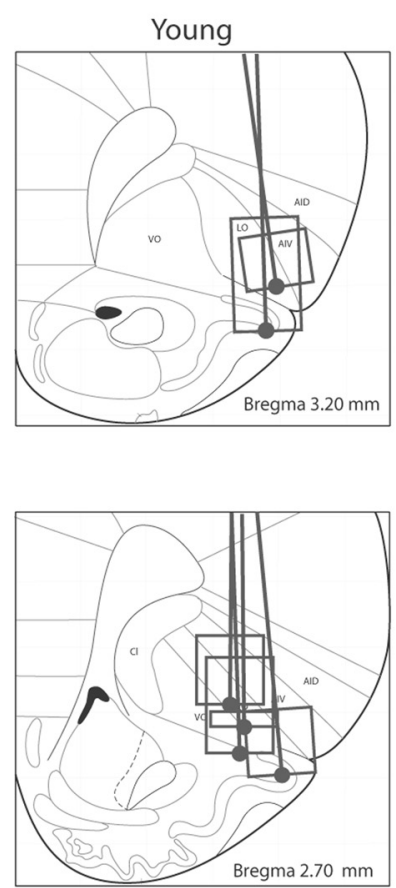

Recording Sites
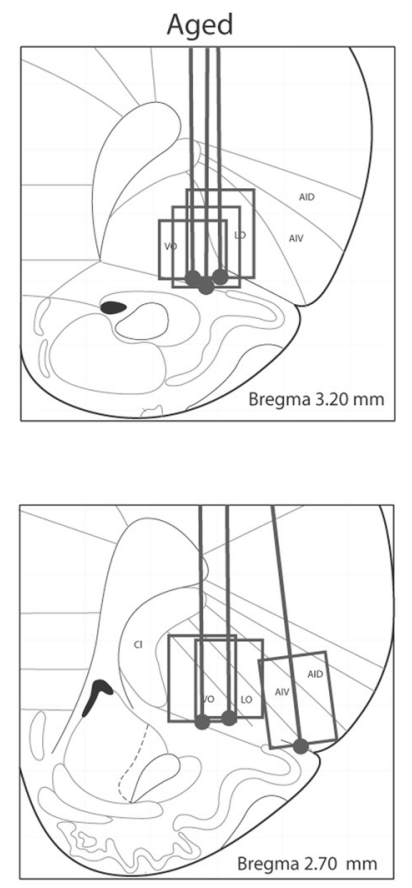

b
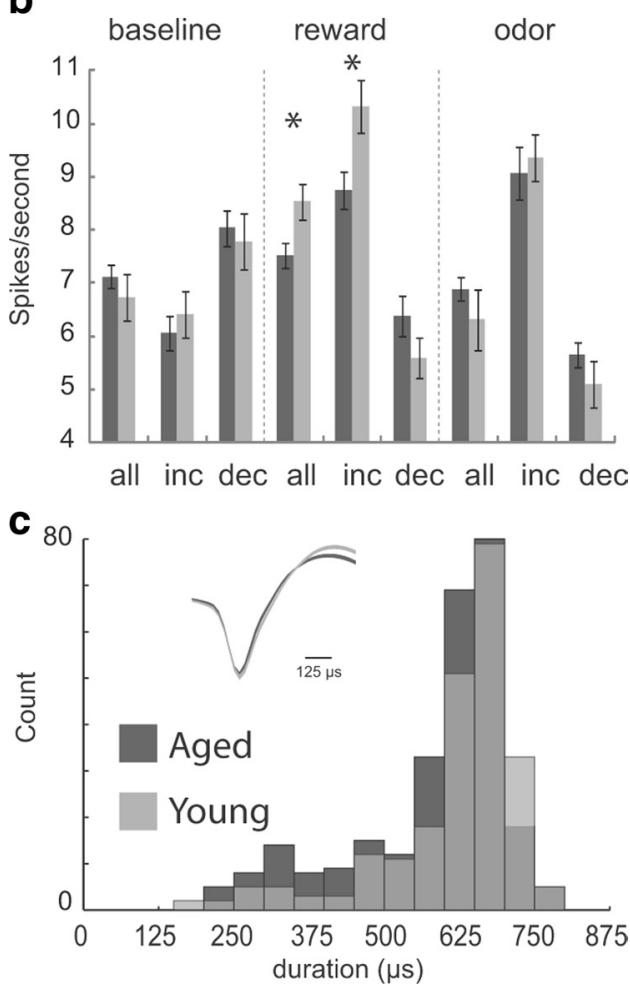

Figure 3. Recording sites and characterization of firing. $\boldsymbol{a}$, Boxes represent the extent of recording locations. Vertical bars on the drawing indicate the center of the electrode track in each rat; boxes indicate approximate extent of recording sessions vertically and give an estimate of lateral (and anterior-posterior) spread of the wires ( $\sim 1 \mathrm{~mm}$ ). $\boldsymbol{b}$, Average firing rate for all neurons and for neurons that showed significant increases (inc) or decreases (dec) in firing during the reward epoch (1 s interval following reward) and the odor epoch (odor onset to port exit) during performance of the task for correct trials only. Asterisks indicate significant differences between young and aged rats ( $t$ test; $p<0.05$ ). Error bars represent $S E M$. Dark and light gray bars represent aged and young groups, respectively. c, Distribution of waveform durations as defined by the time between two maximum amplitudes. Waveforms were not inverted. Inset plots the average waveform for populations of neurons that showed stronger firing on contralateral go trials (black) and stop trials (gray). Shaded area indicates SEM.

forced choice trials, however when given a choice between immediate and delayed reward, aged rats were more willing to wait.

\section{Impact of age on firing in OFC}

We recorded 396 and 619 OFC neurons in young $(n=6)$ and old $(n=6)$ rats, respectively. Baseline firing did not significantly differ between the two groups (Fig. 3b, 5.3 spikes/second versus 5.7 spikes/second, $t$ test; $p=0.50$ ), however waveform durations were significantly reduced in aged rats (Fig. $3 c, t$ test; $p<0.01$ ). In young rats, 229 neurons (58\%) increased firing during the $1 \mathrm{~s}$ following reward delivery compared with baseline (1 s interval before nosepoke; $t$ test; $p<0.05$ ). This population was significantly reduced in aged rats, where only 187 neurons $(30 \%)$ showed reward-evoked responses $\left(\chi^{2}=30.1 ; p<0.01\right)$. Of the neurons that did show reward-evoked responses, the overall firing rate, averaged over the $1 \mathrm{~s}$ after reward delivery was significantly lower in aged compared with young controls (Fig. $3 b, t$ test; $p<0.01)$.

The low responsiveness of OFC neurons in aged rats appeared to be specific to reward-related activity. Odor-evoked responding remained intact (Fig. 3b; odor). In young and aged rats, 110 $(28 \%)$ and 167 neurons (27\%) increased firing during odor sampling (odor onset to odor port exit) compared with baseline ( $1 \mathrm{~s}$ interval before nosepoke; $t$ test; $p<0.05)$, respectively $\left(\chi^{2}=\right.$ $0.02 ; p=0.89)$. The overall average firing rate taken during odor sampling was not significantly different between the two groups (Fig. $3 b ; t$ test; $p=0.71$ ).

\section{Impact of age on reward-related activity}

As described previously, many reward-responsive OFC neurons exhibited firing that was selective for delay or size. This is illustrated in the three single-cell examples in Figure 4, which are aligned on reward onset and show reward-selective activity depending on whether the rat had to wait a short or long time to obtain reward (Fig. $4 a, b$ ) or if the received reward was large or small (Fig. 4c).

The influence of reward size and timing were also evident in the population response of the reward-responsive neurons (Fig. $5 a ; n=229)$. In young rats, activity across the population of reward-responsive neurons increased after well entry until reward delivery on short-delay, small-reward and big-reward trials. Delivery of the second bolus of reward on large-reward trials (Fig. $5 a$; green) prolonged and increased firing in OFC compared with small-reward trials (Fig. $5 a$; orange). For long-delay trials, activity was sustained across the delay, gradually increasing, until delayed reward was delivered. Notably, firing during the delay and at the time of reward delivery on long-delay trials was significantly less compared with firing during delivery of immediate reward (Fig. $5 a$; red vs blue; reward epoch; $t$ test; $p<0.01$ ). Thus, activity in young rats appeared to be stronger for large-reward and short-delay conditions.

To further quantify the influence of reward size and timing, we computed for each neuron the average firing rate for the $1 \mathrm{~s}$ after reward delivery and compared firing on short- versus longdelay and big- vs small-reward trials, respectively. As expected 


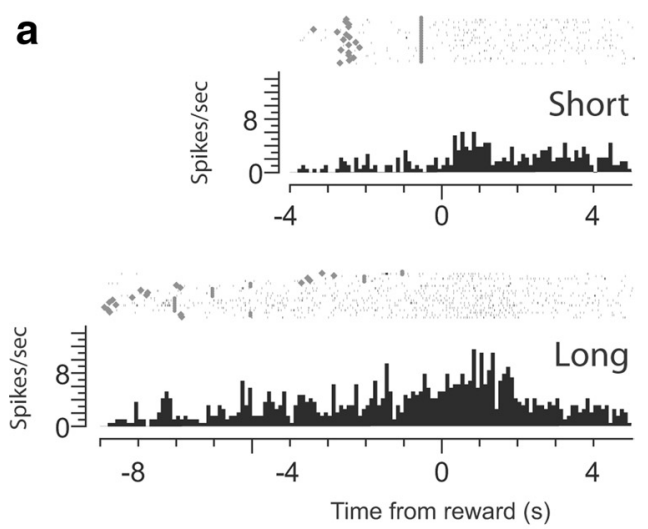

b
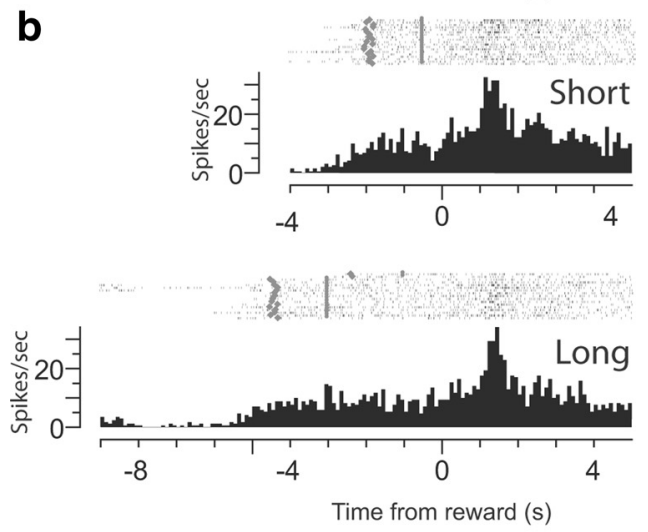

C
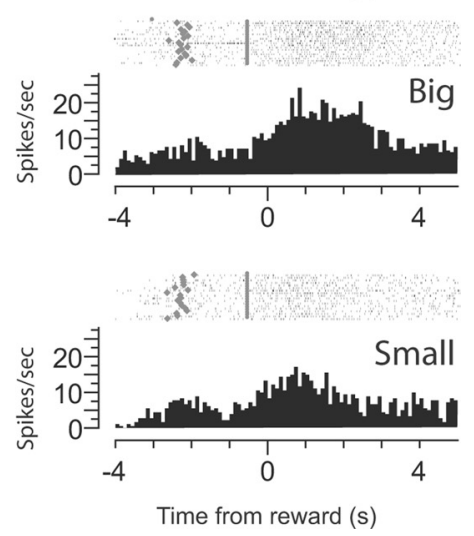

Figure 4. Single-cell examples aligned to onset of reward. $\boldsymbol{a}$, Example of an OFC neuron that increases firing across the delay to reward delivery. Activity was strong for delayed versus more immediate reward. $\boldsymbol{b}$, Example of an OFC neuron that fires more strongly for reward delivered after a short delay. c, Example of an OFC neuron that fires more strongly when the large reward is delivered. Each tick mark represents an action potential. Diamonds, Nosepoke; circles, fluid well entry.

from the population histogram, young rats had significantly more neurons that fired on big-reward and short-delay trials than on small-reward and long-delay trials, respectively $\left(\chi^{2}\right.$ values $>39$; $p$ values $<0.01$; Fig. $5 b)$. Distributions representing the contrast in firing between high and low value outcomes during delay and size blocks were both shifted significantly above zero (Fig. $5 b$; Wilcoxon; $p$ values $<0.01$ ), indicating stronger firing for big-reward and short-delay conditions. However as in our prior report, there was no correlation between size and delay effects. Thus, activity in OFC in young rats appears to maintain separate representations based on the size and timing of reward, and these representations appear to parallel the rats' behavior.

Applying the same analysis to reward responsive neurons in older rats revealed significant differences in firing to delayed reward. Specifically, while the overall proportion of rewardresponsive neurons showing differential firing based on delay did not differ between young and aged rats (69\% versus $60 \% ; \chi^{2}=$ $0.52 ; p=0.47)$, the aged rats did not show the same overrepresentation of the immediate reward that was observed in young rats. Instead, approximately equal numbers of neurons fired to the delayed $(n=80)$ versus the immediate reward $(n=$ $85 ; \chi^{2}=0.14 ; p=0.70$; Fig. $5 d$ ), and the distribution of the delay indices across all neurons was not significantly shifted away from zero (Fig. $5 d$; Wilcoxon; $p$ values $>0.38$ ). This pattern differed significantly from that exhibited by young rats, which showed 117 and 40 neurons that fired significantly more strongly for short and long delayed reward, respectively $\left(\chi^{2}=17.2 ; p<0.01\right)$ and the distribution of delay indices for young (Fig. $5 b$ ) and aged (Fig. $5 d$ ) were significantly different (Wilcoxon; $p<0.01$ ). This balanced representation was also evident in the population response of these neurons, which was approximately the same to reward delivered immediately after a response or several seconds later (Fig. $5 c$ ).

This effect was restricted to delayed reward and did not show up in size blocks. Instead, as in young rats, OFC neurons in aged rats fired more to big than small rewards (Fig. 5c). Thus the number of neurons that fired significantly more for large reward in aged rats was significantly larger than the number that preferred small reward $\left(\chi^{2}=10.8 ; p<0.01\right.$; Fig. $\left.5 c\right)$, and the reward size distributions were shifted significantly above zero (Fig. $5 d$ ). Further, the proportions of neurons exhibiting increased firing to large versus small reward were not significantly different between young and aged rats $\left(\chi^{2}=\right.$ 2.54; $p=0.11$ ).

Although waveform duration and average firing rates of reward-responsive neurons did differ significantly between young and aged rats, this was not the source of the abnormal delay encoding described above. To illustrate this point, we reexamined responding to rewards for neurons in aged rats that had longer waveform durations or higher firing rates compared with their respective counterparts in young rats. The effects described in Figure 5 did not change. For neurons that had a waveform duration that was longer that the mean obtained in young rats, the size index distribution was significantly shifted (Wilcoxon; $p<0.01)$ and the number of cells that significantly increased firing for big and small, was 52 and 17 , respectively $\left(\chi^{2} ; p<\right.$ 0.0001 ). For delay, the distribution was not shifted (Wilcoxon; $p=0.54)$ and the number of cells that significantly increased firing for short and long, was 63 and 55 , respectively $\left(\chi^{2} ; p=\right.$ $0.46)$. The same results were obtained for neurons from aged rats with reward firing that was higher than the mean obtained in young rats. The size index distribution was significantly shifted in the positive direction (Wilcoxon; $p<0.01$ ) and the number of cells that significantly increased firing for big and small rewards, was 27 and 12, respectively $\left(\chi^{2} ; p<0.02\right)$. For delay, the distribution was not shifted (Wilcoxon; $p=0.39$ ) and the number of cells that significantly increased firing for short and long, was 26 and 23 , respectively $\left(\chi^{2} ; p=0.67\right)$.

\section{Impact of age on cue-related activity}

The influence of delay was also evident in the population of the odor-responsive neurons. In young rats, activity across the population of odor-responsive neurons increased after port entry and differentiated odors that predicted differently delayed rewards (Fig. $6 a$; blue versus red). To quantify the influence of 

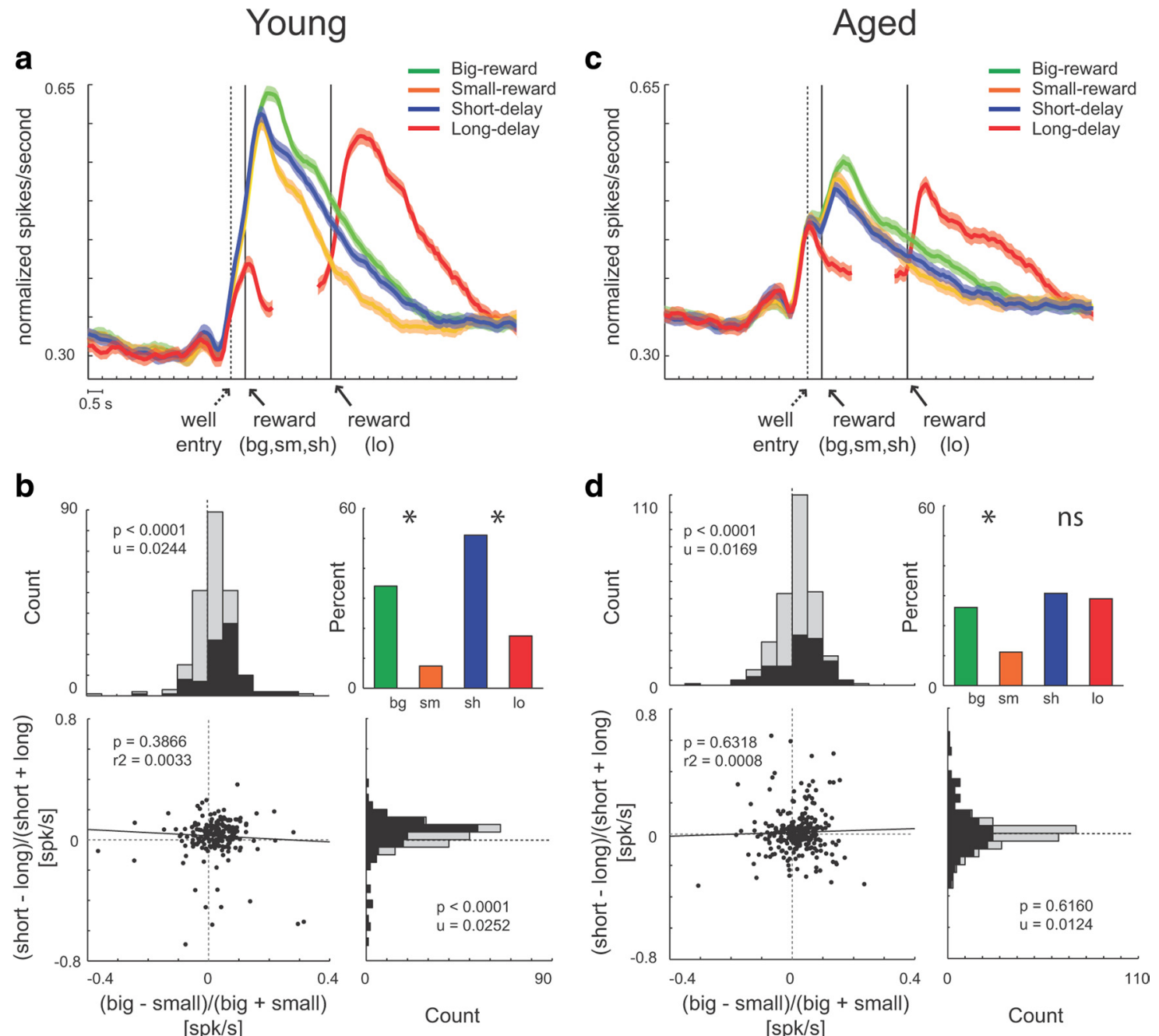

Figure 5. a,c, Plots that average normalized firing rate over each reward-responsive OFC neuron for young and aged populations. For short-delay, big-reward, and small-reward trials, reward was always delivered after $500 \mathrm{~ms}$, thus activity is aligned to both well entry and reward delivery. Since reward delivery on long-delay trials was delayed by $1-7 \mathrm{~s}$ (see Materials and Methods), activity after reward delivery under long-delay trials (red) is broken into two portions, aligned to well entry and reward delivery so that long-delay conditions can be better compared with the other three conditions. Shaded areas represent SEM. $\boldsymbol{b}, \boldsymbol{d}$, Distribution of value indices [delay index $=$ short - long/short + long and size index $=$ big - small/big + small] in young $(\boldsymbol{b})$ and aged $(\boldsymbol{d})$ rats representing the difference in firing (1 sinterval following reward) when high- and low-value outcomes were expected. Significance measured by Wilcoxon. Black and colored bars represent neurons

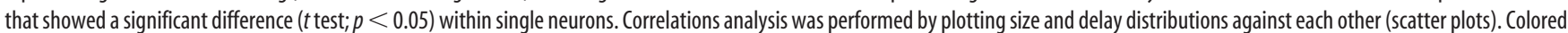
bar graph represents percentage of neurons that fired more strongly for short, long, big, and small average firing during the reward epoch. Asterisks, $\left.\chi^{2} ; p<0.05\right)$.

reward size and timing on firing during odor sampling, we computed for each neuron the average firing rate from odor onset to odor port exit and compared firing on short- versus long-delay and big- vs small-reward trials, respectively. Although the counts of neurons that showed a significant increase in firing for an immediate reward was in the majority ( 31 vs $16 ; \chi^{2}=4.72 ; p<$ 0.05 ; Fig. $6 b$ ), the distributions reflecting the difference between firing during short- and long-delay trials was not significantly shifted from zero (Fig. $6 b$; Wilcoxon; $p=0.19$ ). None of these comparisons were significant for odors that predicted large and small reward (Fig. 6b).

Applying the same analysis to odor responsive neurons in older rats also revealed differences in firing to cues that predicted differently delayed or sized rewards (Fig. $6 c, d$ ). However, in aged rats, there were no significant differences between firing to odors that predicted differently valued rewards in either the size or delay blocks; neither value index was significantly shifted from zero (Wilcoxon; $p$ values $>0.14$ ), and there were equal counts of neurons that fired more strongly for high and low value rewards (Fig. $6 d$; black bars; $\chi^{2}$ values $<1.11$; $p$ values $>0.29$ ).

\section{Discussion}

Here we have shown a relationship between aging-related changes in intertemporal choice (choices between differently delayed rewards) and encoding of delayed rewards in the OFC. Aged rats exhibited a greater propensity to wait for a delayed reward than young controls. Consistent with a prior report on effects of aging on intertemporal choice (Simon et al., 2010), this effect did not reflect changes in responding based on the general value of the reward, since aged rats exhibited normal choices between big and small rewards, nor did the effect reflect an inability to perceive delay of reward, since aged and young rats showed equivalent shifts in accuracy and latency of responding on forced choice trials upon delay of reward. The behavior of aged rats both here and in the earlier study is similar to that of rats with orbitofrontal lesions, which typically show changes in intertemporal choice but not in choices based on reward magnitude (Winstanley et al., 2004). Notably this specific increase in how often the rats waited for the delayed reward was accompanied by an equally specific increase in the relative representation of the delayed reward in the OFC. 

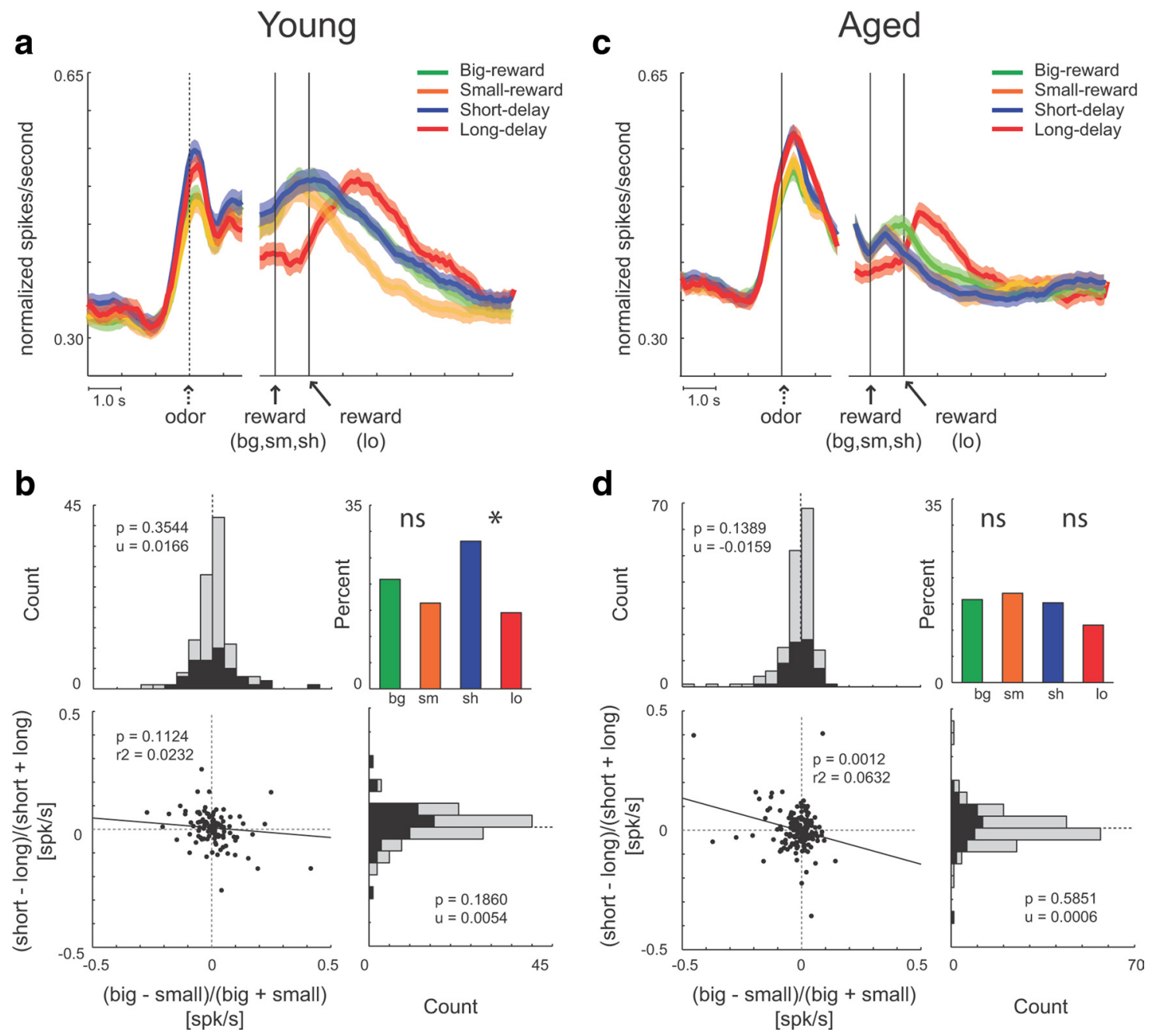

Figure 6. a, c, Plots that average normalized firing rate over each odor-responsive OFC neuron for young and aged populations. Activity is aligned to odor onset and reward delivery. Distribution of value indices [delay index $=$ short - long/short + long and size index $=\mathrm{big}-$ small/ $/ \mathrm{big}+$ small] in young $(\boldsymbol{b})$ and aged $(\boldsymbol{d})$ rats representing the difference in firing (odor onset to odor port exit) when high- and low-value outcomes were expected. Significance measured by Wilcoxon. Black and colored bars represent neurons firing that showed a significant difference $(t$ test; $p<0.05$ ) within single neurons. Correlations analysis was performed by plotting size and delay distributions against each other (scatter plots). Colored bar graph represents percentage of neurons that fired more strongly for short, long, big, and small average firing during the odor epoch. Asterisks: $\chi^{2} ; p<0.05$ ).

These results suggest that aging is associated with changes in orbitofrontal function. Consistent with this, aged rats exhibit deficits in reversal and delayed-non-matching tasks that closely resemble deficits caused by orbitofrontal damage (Otto and Eichenbaum, 1992; Zyzak et al., 1995; Schoenbaum et al., 2002, 2006). Similar impairments have been reported in elderly patients (Lamar and Resnick, 2004; Lamar et al., 2004), and in rats, reversal deficits are linked to altered encoding in orbitofrontal neurons (Schoenbaum et al., 2006).

Yet while these effects are often conceptualized as impairments, normal aging is clearly not equivalent to a lesion, and deficits in orbitofrontal-dependent behaviors are not always observed. When large groups are tested, many elderly rats and human subjects perform normally on tests of orbitofrontal function (Schoenbaum et al., 2002, 2006; Lamar and Resnick, 2004; Lamar et al., 2004), and in some settings, there seem to be no deficits at all. In our own hands, this has been evident in testing for reinforcer devaluation effects. This behavioral task provides a specific assay of the ability to use information about the current value of a reward to guide behavior (Holland and Rescorla, 1975), and it is highly sensitive to orbitofrontal damage (Gallagher et al., 1999;
Pickens et al., 2003; Izquierdo et al., 2004). Yet aged rats performed as well or better than young controls at adjusting behavior in this context (Singh et al., 2011).

Results such as these suggest a more nuanced view of the changes in how information is encoded that come with normal aging, at least in the OFC. Altered encoding may change behavior for the better or for the worse, depending on the needs of the task at hand. In the task used here, the time between consecutive trials and the total reward received, which are objective determinants of total reward value, were held constant over delay blocks independent of choice performance; thus, the enhanced encoding and willingness to wait observed in aged rats over controls could reflect a relative increase in the perceived, subjective value of the delayed reward. In contrast, the total reward received during size blocks was directly determined by choice performance; similar performance suggests that aged rats were just as adept as young rats at actually obtaining reward in this task. It follows that under a delay discounting paradigm where choices may reflect the total reward received that age may not be a determinant of behavior.

In this regard, it is also worth noting that while the OFC is involved in both reversal learning, devaluation, and discounting, 
current hypotheses would suggest that its involvement in each reflects potentially different functions and circuits. While its role in devaluation almost certainly involves the use of existing information, prospectively, to guide behavior, its role in reversal learning likely reflects the use of that same or similar information, retrospectively, to facilitate accurate updating of associative information previously (Schoenbaum et al., 2009; Walton et al., 2010). Thus the effect of aging-related changes in orbitofrontal representations might depend on whether these representations are necessary for directing behavior, in which case there seems to be little effect or perhaps even enhanced function; or for learning, in which case there seem to be impairment. Interestingly discounting likely requires both functions-the former to guide choices on the current trial and the latter to update representations as a reward is delayed, and thus less valuable, to affect behavior on future trials. One possible interpretation of the current neurophysiological results, which show an over-representation of the delayed reward in the OFC, is that aging preserves the representation of reward expectancies while perhaps disrupting the ability of these representations to support learning.

Of course, delay of reward affects neural activity in numerous other brain regions. Indeed, as noted earlier, the OFC is just part of a circuit of structures implicated specifically in discounting delayed rewards (Cousins et al., 1996; Cardinal et al., 2001, 2004; Mobini et al., 2002; Winstanley et al., 2004; Cardinal, 2006; Rudebeck et al., 2006; Bezzina et al., 2007; Winstanley, 2007; Floresco et al., 2008; Kalenscher and Pennartz, 2008; Churchwell et al., 2009; Ghods-Sharifi et al., 2009; Sellitto et al., 2010; Zeeb et al., 2010; Mar et al., 2011). It is impossible to say with certainty whether changes in neural correlates, such as those demonstrated here, reflect local changes in how incoming information is processed or broader changes elsewhere within the circuit that alter input to OFC. For example, both amygdala and ventral striatum have been implicated in discounting, and while both are typically viewed as "downstream" of the OFC, both would be capable of influencing activity in the OFC via either direct or indirect projections. Thus aging-related changes in representations in OFC could reflect alterations in how these other parts of the circuit are processing information. A program combining behavioral and recording approaches in both young and aged animals to delineate how information flows between these regions, combined with behaviorally guided studies of molecular and neurophysiological changes at the cellular level, would be necessary to address this deeper question.

\section{References}

Ainslie GW (1974) Impulse control in pigeons. J Exp Anal Behav 21:485-489.

Barense MD, Fox MT, Baxter MG (2002) Aged rats are impaired on an attentional set-shifting task sensitive to medial frontal cortex damage in young rats. Learn Mem 9:191-201.

Bezzina G, Cheung TH, Asgari K, Hampson CL, Body S, Bradshaw CM, Szabadi E, Deakin JF, Anderson IM (2007) Effects of quinolinic acidinduced lesions of the nucleus accumbens core on inter-temporal choice: a quantitative analysis. Psychopharmacology (Berl) 195:71-84.

Brushfield AM, Luu TT, Callahan BD, Gilbert PE (2008) A comparison of discrimination and reversal learning for olfactory and visual stimuli in aged rats. Behav Neurosci 122:54-62.

Cai X, Kim S, Lee D (2011) Heterogeneous coding of temporally discounted values in the dorsal and ventral striatum during intertemporal choice. Neuron 69:170-182.

Cardinal RN (2006) Neural systems implicated in delayed and probabilistic reinforcement. Neural Netw 19:1277-1301.

Cardinal RN, Pennicott DR, Sugathapala CL, Robbins TW, Everitt BJ (2001) Impulsive choice induced in rats by lesions of the nucleus accumbens core. Science 292:2499-2501.
Cardinal RN, Winstanley CA, Robbins TW, Everitt BJ (2004) Limbic corticostriatal systems and delayed reinforcement. Ann N Y Acad Sci 1021:33-50

Churchwell JC, Morris AM, Heurtelou NM, Kesner RP (2009) Interactions between the prefrontal cortex and amygdala during delay discounting and reversal. Behav Neurosci 123:1185-1196.

Cousins MS, Atherton A, Turner L, Salamone JD (1996) Nucleus accumbens dopamine depletions alter relative response allocation in a T-maze cost/benefit task. Behav Brain Res 74:189-197.

Day JJ, Jones JL, Wightman RM, Carelli RM (2010) Phasic nucleus accumbens dopamine release encodes effort- and delay-related costs. Biol Psychiatry 68:306-309.

Day JJ, Jones JL, Carelli RM (2011) Nucleus accumbens neurons encode predicted and ongoing reward costs in rats. Eur J Neurosci 33:308-321.

Evenden JL, Ryan CN (1996) The pharmacology of impulsive behaviour in rats: the effects of drugs on response choice with varying delays of reinforcement. Psychopharmacology (Berl) 128:161-170.

Floresco SB, St Onge JR, Ghods-Sharifi S, Winstanley CA (2008) Corticolimbic-striatal circuits subserving different forms of cost-benefit decision making. Cogn Affect Behav Neurosci 8:375-389.

Gallagher M, McMahan RW, Schoenbaum G (1999) Orbitofrontal cortex and representation of incentive value in associative learning. J Neurosci 19:6610-6614.

Gazzaley A, Cooney JW, Rissman J, D’Esposito M (2005) Top-down suppression deficit underlies working memory impairment in normal aging. Nat Neurosci 8:1298-1300.

Ghods-Sharifi S, Floresco SB (2010) Differential effects on effort discounting induced by inactivations of the nucleus accumbens core or shell. Behav Neurosci 124:179-191.

Ghods-Sharifi S, St Onge JR, Floresco SB (2009) Fundamental contribution by the basolateral amygdala to different forms of decision making. J Neurosci 29:5251-5259.

Herrnstein RJ (1961) Relative and absolute strength of response as a function of frequency of reinforcement. J Exp Anal Behav 4:267-272.

Holland PC, Rescorla RA (1975) The effects of two ways of devaluing the unconditioned stimulus after first and second-order appetitive conditioning. J Exp Psychol Anim Behav Process 1:355-363.

Izquierdo A, Suda RK, Murray EA (2004) Bilateral orbital prefrontal cortex lesions in rhesus monkeys disrupt choices guided by both reward value and reward contingency. J Neurosci 24:7540-7548.

Kalenscher T, Pennartz CM (2008) Is a bird in the hand worth two in the future? The neuroeconomics of intertemporal decision-making. Prog Neurobiol 84:284-315.

Kesner RP, Williams JM (1995) Memory for magnitude of reinforcement: dissociation between amygdala and hippocampus. Neurobiol Learn Mem 64:237-244.

Lamar M, Resnick SM (2004) Aging and prefrontal functions: dissociating orbitofrontal and dorsolateral abilities. Neurobiol Aging 25:553-558.

Lamar M, Yousem DM, Resnick SM (2004) Age differences in orbitofrontal activation: an fMRI investigation of delayed match and nonmatch to sample. Neuroimage 21:1368-1376.

Lasarge CL, Bañuelos C, Mayse JD, Bizon JL (2009) Blockade of GABA(B) receptors completely reverses age-related learning impairment. Neuroscience 164:941-947.

Mar AC, Walker AL, Theobald DE, Eagle DM, Robbins TW (2011) Dissociable effects of lesions to orbitofrontal cortex subregions on impulsive choice in the rat. J Neurosci 31:6398-6404.

Marschner A, Mell T, Wartenburger I, Villringer A, Reischies FM, Heekeren HR (2005) Reward-based decision-making and aging. Brain Res Bull 67:382-390.

McDannald MA, Lucantonio F, Burke KA, Niv Y, Schoenbaum G (2011) Ventral striatum and orbitofrontal cortex are both required for modelbased, but not model-free, reinforcement learning. J Neurosci 31:2700-2705.

Mizoguchi K, Shoji H, Tanaka Y, Maruyama W, Tabira T (2009) Agerelated spatial working memory impairment is caused by prefrontal cortical dopaminergic dysfunction in rats. Neuroscience 162:1192-1201.

Mobini S, Body S, Ho MY, Bradshaw CM, Szabadi E, Deakin JF, Anderson IM (2002) Effects of lesions of the orbitofrontal cortex on sensitivity to delayed and probabilistic reinforcement. Psychopharmacology (Berl) 160:290-298.

Moore TL, Killiany RJ, Herndon JG, Rosene DL, Moss MB (2006) Executive 
system dysfunction occurs as early as middle-age in the rhesus monkey. Neurobiol Aging 27:1484-1493.

Otto T, Eichenbaum H (1992) Complementary roles of the orbital prefrontal cortex and the perirhinal-entorhinal cortices in an odor-guided delayed-nonmatching-to-sample task. Behav Neurosci 106:762-775.

Pickens CL, Saddoris MP, Setlow B, Gallagher M, Holland PC, Schoenbaum G (2003) Different roles for orbitofrontal cortex and basolateral amygdala in a reinforcer devaluation task. J Neurosci 23:11078-11084.

Rachlin H, Green L (1972) Commitment, choice and self-control. J Exp Anal Behav 17:15-22.

Rapp PR, Heindel WC (1994) Memory systems in normal and pathological aging. Curr Opin Neurol 7:294-298.

Roesch MR, Taylor AR, Schoenbaum G (2006) Encoding of timediscounted rewards in orbitofrontal cortex is independent of value representation. Neuron 51:509-520.

Roesch MR, Singh T, Brown PL, Mullins SE, Schoenbaum G (2009) Ventral striatal neurons encode the value of the chosen action in rats deciding between differently delayed or sized rewards. J Neurosci 29:13365-13376.

Rudebeck PH, Walton ME, Smyth AN, Bannerman DM, Rushworth MF (2006) Separate neural pathways process different decision costs. Nat Neurosci 9:1161-1168.

Rypma B, D'Esposito M (2000) Isolating the neural mechanisms of agerelated changes in human working memory. Nat Neurosci 3:509-515.

Salinas JA, Packard MG, McGaugh JL (1993) Amygdala modulates memory for changes in reward magnitude: reversible post-training inactivation with lidocaine attenuates the response to a reduction in reward. Behav Brain Res 59:153-159.

Schoenbaum G, Nugent S, Saddoris MP, Gallagher M (2002) Teaching old rats new tricks: age-related impairments in olfactory reversal learning. Neurobiol Aging 23:555-564.

Schoenbaum G, Setlow B, Saddoris MP, Gallagher M (2006) Encoding changes in orbitofrontal cortex in reversal-impaired aged rats. J Neurophysiol 95:1509-1517.

Schoenbaum G, Roesch MR, Stalnaker TA, Takahashi YK (2009) A new perspective on the role of the orbitofrontal cortex in adaptive behaviour. Nat Rev Neurosci 10:885-892.

Sellitto M, Ciaramelli E, di Pellegrino G (2010) Myopic discounting of future rewards after medial orbitofrontal damage in humans. J Neurosci 30:16429-16436.

Simon NW, LaSarge CL, Montgomery KS, Williams MT, Mendez IA, Setlow B, Bizon JL (2010) Good things come to those who wait: attenuated discounting of delayed rewards in aged Fischer-344 rats. Neurobiol Aging 31:853-862.

Singh T, Jones JL, McDannald MA, Haney RZ, Cerri DH, Schoenbaum G (2011) Normal aging does not impair orbitofrontal-dependent reinforcer devaluation effects. Front Aging Neurosci 3:4.

Smith DE, Rapp PR, McKay HM, Roberts JA, Tuszynski MH (2004) Memory impairment in aged primates is associated with focal death of cortical neurons and atrophy of subcortical neurons. J Neurosci 24:4373-4381.

Walton ME, Behrens TEJ, Buckley MJ, Rudebeck PH, Rushworth MFS (2010) Separable learning systems in the macaque brain and the role of the orbitofrontal cortex in contingent learning. Neuron 65:927-939.

Wang M, Gamo NJ, Yang Y, Jin LE, Wang XJ, Laubach M, Mazer JA, Lee D, Arnsten AF (2011) Neuronal basis of age-related working memory decline. Nature 476:210-213.

Winstanley CA (2007) The orbitofrontal cortex, impulsivity, and addiction: probing orbitofrontal dysfunction at the neural, neurochemical, and molecular level. Ann N Y Acad Sci 1121:639-655.

Winstanley CA, Theobald DEH, Cardinal RN, Robbins TW (2004) Contrasting roles of basolateral amygdala and orbitofrontal cortex in impulsive choice. J Neurosci 24:4718-4722.

Zeeb FD, Floresco SB, Winstanley CA (2010) Contributions of the orbitofrontal cortex to impulsive choice: interactions with basal levels of impulsivity, dopamine signalling, and reward-related cues. Psychopharmacol (Berl) 211:87-98.

Zyzak DR, Otto T, Eichenbaum H, Gallagher M (1995) Cognitive decline associated with normal aging in rats: a neuropsychological approach. Learn Mem 2:1-16. 